\title{
Evaluation of rational antibiotic use in simple traumatic wounds (STWs) based on Thailand core indicators in database of university hospital
}

Patawee Detchit ${ }^{1}$,

Narathan Thamjitsakul ${ }^{1}$, Thanaphoom Kiewchaum ${ }^{1}$, Tanapong Chainakul2, Nantawarn Kitikannakorn ${ }^{*}$

1 Faculty of Pharmacy, Chiangmai University, Muang, Chiangmai, Thailand 2 Maharaj Nakhon Chiangmai Hospital, Faculty of Medicine, Chiangmai University, Muang, Chiangmai, Thailand

*Corresponding author: Nantawarn Kitikannakorn nantawarn.k@cmu.ac.th

Keywords:

Rational drug use;

Simple traumatic wound;

Antibiotics; Antibiotic smart use

https://www.pharmacy.mahidol.ac.th/journal/ (C) Faculty of Pharmacy, Mahidol University (Thailand) 2021

\begin{abstract}
In Thailand, Ministry of Public Health (MoPH) enforces core rational drug use (RDU) indicators in hospital. Antibiotics for simple traumatic wounds (STWs) cases, one of rational antibiotic indicator for out-patient department (OPD), must less than $40 \%$ of all cases. This study aims to evaluate antibiotic use in STWs cases from database of the university hospital. OPD service data are gathered during January to November 2018. We include totally 13,566 medical records which stated ICD-10 code of STWs through phpMyAdmin program and an entity-relationship (E-R) diagram. Followed the manual, non-rational prescribing of oral and injection antibiotics (ATC-ID code: J01) are 2,549 times (18.8\%). It somehow achieves target of MoPH's indicator. From literature review and expert's opinion, some types of STWs need antibiotics for treatment. When we exclude necessary cases, non-rational antibiotic use is only $11.4 \%$. Beside quantitative data, we explore a list of prescribed antibiotics. A number of drugs is not in accordance with standard guidelines e.g. norfloxacin, ofloxacin, co-trimoxazole, clindamycin, moxifloxacin, and roxithromycin. Inadequate dose and duration of treatment are detected. Amoxicillin-clavulanic acid is the most frequently prescribed, which is not a drug of choice and high cost. Injection antibiotic, such as ertapenem, at OPD is not appropriate when oral formulations are available. Yearly cost of antibiotics for STWs is 274,275 Thai Baht, $21.00 \%$ of this cost is non-rational use. This study reflects wastage of scarce resources and leading to increased risk of antimicrobial resistance. Further study needs more information to improve irrational items, dose, range, and route of antibiotics.
\end{abstract}

\section{INTRODUCTION}

World Health Organization (WHO) estimates that more than half of all drugs are irrationally prescribed, and more than half of the patients fail to adhere the prescribed regimens. Rational drug use (RDU) generally aims to promote appropriate prescribing medicines for diseases and health conditions together with cost-effective and community affordable ${ }^{1}$. In Thailand, Ministry of public health (MoPH) announces RDU manual to monitor core indicators for quantitative evaluation of rational drug use in hospital ${ }^{2}$. RDU manual does major purpose for assisting practitioners in rational clinical decision-making. In case of infectious diseases, routine approach of manual is often in conflict with the concept of personalized medicine and shared decision-making. Assessment qualitative of care through antibiotic use from hospital 
database is somewhat additional interesting. We need to discover details of regimen, route of administration, duration of treatment, cost of drug therapy, preventable adverse drug reactions, drug interactions, and patient adherence to maximize therapeutic outcomes ${ }^{1,3}$.

In previous study, we conducted LibreOffice program and an entity-relationship (E-R) diagram as a tool for RDU evaluation. Results of upper respiratory infections (URIs) and acute diarrhea (AD) cases showed both quantitative of antibiotics use and interesting qualitative data of medication errors and drug related problems. The weak point of the tool was limited amount of input data ${ }^{4}$. Assessment RDU of simple traumatic wounds (STWs), another important core indicator, is waiting to explore by an improvement tool. Over 500 ICD-10 codes of STWs is stated in RDU manual. Antibiotic prescribed must less than $40 \%$ of all STWs cases at out-patient department (OPD) ${ }^{2}$. Nevertheless, too many literatures ${ }^{5-7}$ and expert's opinion suggest that some types of STWs need antibiotics for treatment. Beside quantitative indicators followed RDU manual, this study decided to exclude ICD-10 codes of necessary cases.

This study aims to encounters with percentage prescription of antibiotics and quality of care for STWs from the hospital's database. We use phpMyAdmin program and SQL (Structured Query Language) database which is an open-source software tool. Basically, phpMyAdmin is a tool to manage and manipulating the tables and data inside the database. It mainly purposes to create, update, drop, alter, delete, import, and export data in hospital database without limit in amount of data. SQL database is a domainspecific language used in programming and design for managing data held in a relational database management system. We draw a specific entity-relationship (E-R) diagram that crosses boundaries and enables variables from hospital database to calculate an outcome more easily and gain more productive information.

\section{MATERIALS AND METHODS}

\subsection{Data collection for STWs cases}

We create a tool from phpMyAdmin program and SQL database. It uses to analyze RDU data from hospital's database. The study code D-PHA-2561-05365 is exempt for ethic allowance from the ethical review committee for human research, Faculty of medicine, Chiangmai University on January $17^{\text {th }}, 2019$. All prescription records are collected during January to November 2018. Inclusion criteria were patient who (1) diagnosed for ICD-10 of STWs and (2) prescribed systematic antibiotics (ATC-ID code J01). All ICD-10 of STWs are regulated in "Rational drug use manual for rational drug use in health promotion hospital" 2 . We excluded patient who referred and prescribed antibiotics from other hospitals.

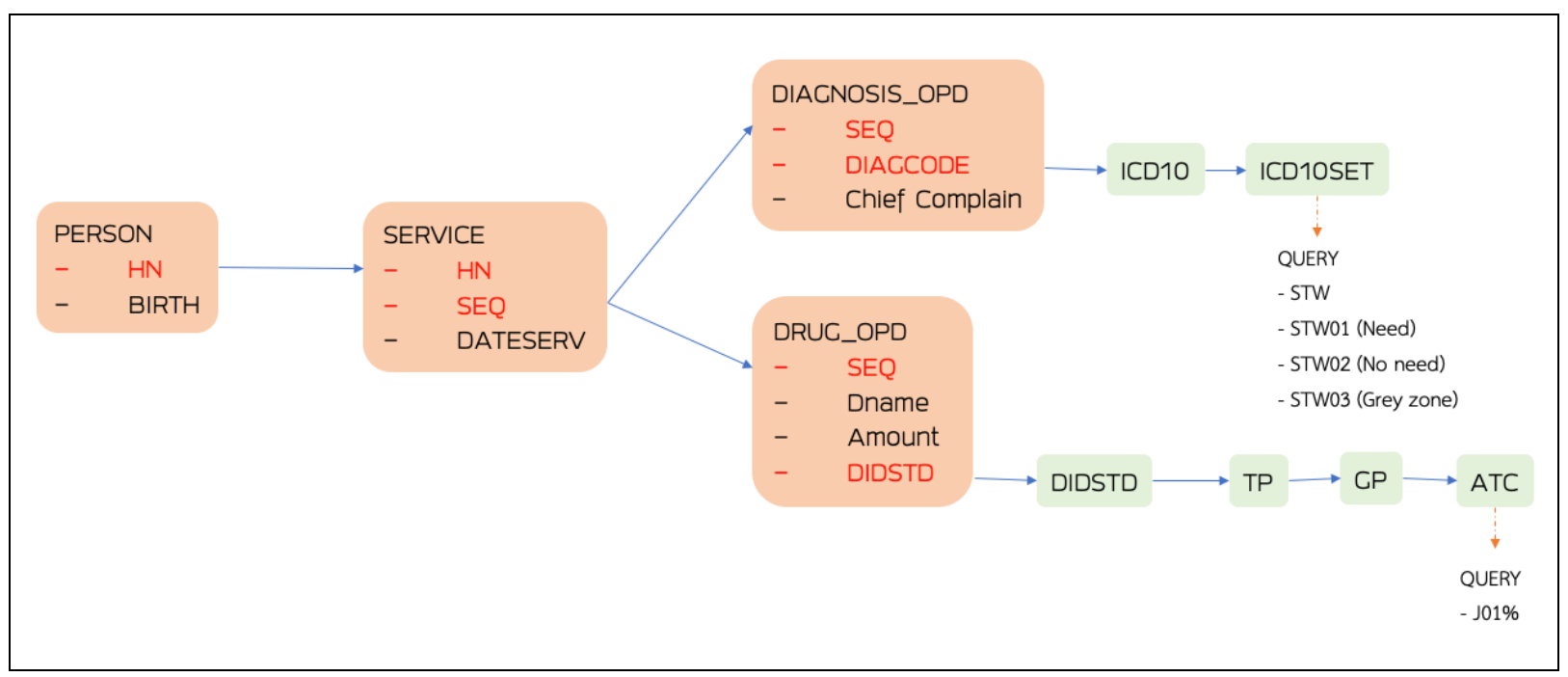

Figure 1. An entity-relationship (E-R) diagram that crosses boundaries and enables variables form hospital database to calculate an outcome more easily. $H N=$ Hospital Number, BIRTH = Birthday, SEQ = Sequence Number, DATESERV = Date Service, DIAGCODE = Diagnosis Code, Dname $=$ Drug Name, DIDSTD $=$ Drug Identification Standard, ICD10 $=$ International Classification of Diseases 10th Revision, STWs = Simple Traumatic Wounds, TP = Trade Product, GP $=$ Generic Product, ATC = Anatomical Therapeutic Chemical code, J01\% = ATC code J01, Antibacterial for Systemic Use in Subgroup of the Anatomical Therapeutic Chemical Classification System 
2.2. Mapping drug code from hospital database to standard research variables

Thai FDA determines outcome of RDU from ICD-10 for diagnosis and ATC-ID for drug $\operatorname{code}^{2}$. But the university hospital database uses DIDSTD (Drug Identification Standard) for local drug code. Consequence, this study does an entity relationship (E-R) diagram as shown in Figure 1. This relationship of entity sets crossing boundaries and enables variables form hospital database to calculate an outcome more easily. We map all codes of systemic antibiotics both orally and injection from DIDSTD code in hospital database to ATC code. Each ATC-ID code is paired to DIDSTD code in hospital database through TPID and GPID. The mapping feature enables to replace requested ATCID from National policy to specific drug for analysis in hospital database (Figure 2). ATC-ID codes, in this study, covered J01GB06，J01CR02，J01CA04，J01CR01，
J01CA01，J01CR51，J01FA10，J01CE08, J01CE01，J01DH05，J01DC04，J01DB01, J01DC03，J01DB04，J01DD15，J01DD16, J01DE01，J01DD08，J01DC12，J01DD62, J01DD12，J01DD01，J01DC01，J01DE02， J01DD02，J01DD14，J01DD04，J01DC02， J01BA01，J01AA03，J01DH51，J01MA02, J01FA09, J01FF01，J01CF02， J01XB01, J01XX09，J01CF01，J01DH04，J01AA02, J01DH03，J01FA01，J01RA02 J01XX01, J01XC01，J01MA16，J01GB03，J01GB04, J01MA12，J01AA56，J01FF02，J01XX08, J01AA04，J01DH02，J01XD01，J01AA08, J01MA14，J01GB07，J01XE01，J01MA06, J01MA01，J01AA06，J01CR50，J01EB20, J01CE02，J01MB04，J01CR05，J01CA12, J01CA02, J01MA17, J01FA06, J01MA10, J01MA21，J01XC01，J01FA02，J01GA01, J01CG01，J01EC02，J01EE02，J01EE01， J01CR04，J01XA02，J01FA15，J01AA07, J01BA02，J01AA12，J01EA01，J01XA01.

\begin{tabular}{|l|r|r|r|}
\hline DIDSTD & TPID & GPID & ATCID \\
\hline 100702000003750120381169 & 163239 & 210120 & M01AB05 \\
\hline 100702000003750120381202 & 163343 & 210120 & M01AB05 \\
\hline 100702000003750121781257 & 163447 & 210120 & M01AB05 \\
\hline 100702000003850121781169 & 164246 & 210183 & M01AB05 \\
\hline 100702000003850120381202 & 164267 & 210183 & M01AB05 \\
\hline 124813000003620120381506 & 154988 & 210448 & C08CA01 \\
\hline 124813000003620120381202 & 155020 & 210448 & C08CA01 \\
\hline 124813000003620120381421 & 155065 & 210448 & C08CA01 \\
\hline 124813000003620120381247 & 155077 & 210448 & C08CA01 \\
\hline 124813000003620120381176 & 155083 & 210448 & C08CA01 \\
\hline 124813000003620120381079 & 155096 & 210448 & C08CA01 \\
\hline 124813000003400121781401 & 155123 & 210453 & C08CA01 \\
\hline 124813000003521121781401 & 155134 & 210469 & C08CA01 \\
\hline 124813000003521120381202 & 155168 & 210476 & C08CA01 \\
\hline 124813000003521120381421 & 155201 & 210476 & C08CA01 \\
\hline 124813000003521120381179 & 155217 & 210476 & C08CA01 \\
\hline
\end{tabular}

Figure 2. Example relation of mapping drug code. ATCID code was paired to DIDSTD code in hospital database through TPID and GPID. The mapping feature enables to replace requested ATCID from National policy to specific drug for analysis in hospital database. DIDSTD $=$ drug identification standard, TPID $=$ Trade product identification, $G P I D=$ Generic product identification, ATCID = Anatomical Therapeutic Chemical code identification

\subsection{Descriptive analysis of rational antibiotic use for STWs}

ICD-10 codes of STWs are S-00S01, S05, S-07S11, S-16S21, S-28S31, S-38S41, S-46 S51, S-56S61, S-66S71, S-76S81, S-86S91, S-96 S99, T-00T01, T-04T07, T-0.09T1.09, T-0.11 T1.11, T-0.13T1.13, T-0.14T1.14, T-6.14T9.14, T-20T25, T-29T32, W-50W64, X-00X19, X-20
X29, X-30X39. Infection risk assessment based on type and location of wound. Then, we classify the ICD-10 codes into 3 groups.

\subsubsection{ICD-10 codes of STWs which need antibiotic}

Antibiotics is likely to be used when the wound was more than 8 hours old, involved a puncture, or involved a skin or subcutaneous fat amputation in emergency departments. Traumatic 
wound from a dog bite also deserves on a caseby-case basis for oral antibiotics, especially in high risk bite wounds (such as extremity wounds) ${ }^{6}$. In accordance with expert's opinion, antibiotics do necessity for open wound at genital area, amputation, crushing wound, bite wounds from mammals ${ }^{3,7}$. In summary, this group of ICD-10 codes take account of S4.05, S5.05, S6.05, S7.05, S0.07, S1.07, S8.07, S9.07, S0.08, S1.08, S8.08, S9.08, S1.09, S2.09, S0.16, S0.17, S8.17, S9.17, S0.28, S1.28, S0.29, S2.31, S3.31, S4.31, S5.31, S0.38, S1.38, S2.38, S3.38, S0.39, S0.46, S1.46, S2.46, S3.46, S7.46, S8.46, S9.46, S0.48, S1.48, S9.48, S7.49, S8.49, S9.49, S0.56, S1.56, S2.56, S3.56, S4.56, S5.56, S7.56, S8.56, S0.57, S8.57, S9.57, S0.58, S1.58, S9.58, S7.59, S8.59, S9.59, S0.66, S1.66, S2.66, S3.66, S4.66, S5.66, S6.66, S7.66, S8.66, S9.66, S0.67, S8.67, S0.68, S1.68, S2.68, S3.68, S4.68, S8.68, S9.68, S7.69, S8.69, S9.69, S0.76, S1.76, S2.76, S3.76, S4.76, S7.76, S0.77, S1.77, S2.77, S0.78, S1.78, S9.78, S7.79, S8.79, S9.79, S1.86, S2.86, S3.86, S7.86, S8.86, S9.86, S0.87, S8.87, S0.88, S1.88, S9.88, S7.89, S8.89, S9.89, S0.96, S1.96, S2.96, S7.96, S8.96, S9.96, S0.97, S1.97, S8.97, S0.98, S1.98, S2.98, S3.98, S4.98, S7.99, S8.99, S9.99, T0.04, T1.04, T2.04, T3.04, T4.04, T7.04, T8.04, T9.04, T0.05, T1.05, T2.05, T3.05, T4.05, T5.05, T6.05, T8.05, T9.05, T4.06,T5.06, T7.14, T6.14, W0.50, W0.53, W0.54, W0.55, W0.58, W0.59.

\subsubsection{ICD-10 codes of STWs which unnecessary antibiotics}

There is no evidence to support antibiotic prophylaxis for superficial injury, bite wound from non-mammal animals, burn, simple laceration, and corrosions ${ }^{5,8}$. Then, this group of ICD-10 codes consist of S0.00, S1.00, S2.00, S3.00, S4.00, S5.00, S7.00, S8.00, S9.00, S1.05, S0.10, S7.10, S8.10, S9.10, S0.20, S1.20, S2.20, S3.20, S4.20, S7.20, S8.20, S0.30, S1.30, S2.30, S7.30, S8.30, S9.30, S0.40, S7.40, S8.40, S9.40, S0.50, S1.50, S7.50, S8.50, S9.50, S0.60, S1.60, S2.60, S7.60, S8.60, S9.60, S0.70, S1.70, S7.70, S8.70, S9.70, S0.80, S1.80, S7.80, S8.80, S9.80, S0.86, S0.90, S1.90, S2.90, S3.90, S7.90, S8.90, S9.90, T0.00, T1.00, T2.00, T3.00, T6.00, T8.00, T9.00, T0.09, T0.11, T0.13, T0.14, T0.20, T1.20, T2.20, T3.20, T4.20, T5.20, T6.20, T7.20, T0.21, T1.21, T2.21, T3.21, T4.21, T5.21, T6.21, T7.21, T0.22, T1.22, T2.22, T3.22, T4.22, T5.22, T6.22, $\mathrm{T} 7.22, \mathrm{~T} 0.23, \mathrm{~T} 1.23, \mathrm{~T} 2.23, \mathrm{~T} 3.23, \mathrm{~T} 4.23, \mathrm{~T} 5.23$, T6.23, T7.23, T0.24, T1.24, T2.24, T3.24, T4.24, T5.24, T6.24, T7.24, T0.25, T1.25, T2.25, T3.25, T4.25, T5.25, T6.25, T7.25, T0.29, T1.29, T2.29,
T3.29, T4.29, T5.29, T6.29, T7.29, T0.30, T1.30, T2.30, T3.30, T4.30, T5.30, T6.30, T7.30, T0.31, T1.31, T2.31, T3.31, T4.31, T5.31, T6.31, T7.31, T8.31, T9.31, T0.32, T.1.32 T2.32, T3.32, T4.32, T5.32, T6.32, T7.32, T8.32, T9.32, W0.52, W0.57, W0.60, W0.64, X0.20, X0.21, X0.22, X0.23, X0.24, X0.25, X0.26, X0.27, X0.28, X0.29.

\subsubsection{ICD-10 codes of STWs which indistinguishable decision to propose antibiotics}

Routine use of prophylactic systemic antibiotics is likely unnecessary and should be considered on a case by case basis. This group is a set of ICD-10 codes of open wound at leg, arm, head, and body. Any traumatic injury of the hand should be considered for a possible tendon injury, especially if located on the volar or dorsal side. ${ }^{7}$ The included ICD-10 codes are S0.01, S1.01, S2.01, S3.01, S4.01, S5.01, S7.01, S8.01, S9.01, S0.05, S2.05, S3.05, S8.05, S9.05, S0.09, S7.09, S8.09, S9.09, S1.10, S0.11, S1.11, S2.11, S7.11, S8.11, S9.11, S7.19, S8.19, S9.19, S0.21, S1.21, S2.21, S7.21, S8.21, S9.21, S7.29, S8.29, S9.29, S0.31, S1.31, S7.31, S8.31, S6.39, S7.39, S8.39, S9.39, S0.41, S1.41, S7.41, S8.41, S0.51, S7.51, S8.51, S9.51, S0.61, S1.61, S7.61, S8.61, S9.61, S0.71, S1.71, S7.71, S8.71, S0.81, S7.81, S8.81, S9.81, S0.91, S1.91, S2.91, S3.91, S7.91, T0.01, T1.01, T2.01, T3.01, T6.01, T8.01, T9.01, T0.06, T1.06, T2.06, T3.06, T8.06, T1.09, T1.11, T1.13, T1.14, T8.14, T9.14. We include elderly (>65 years old) ${ }^{7}$ and immunocompromised population in this high-risk group as well. This group is a set of ICD-10 codes of patients with HIV/AIDS, chemotherapy recipients, organ transplant recipients, vascular diseases and diabetics $^{6,7}$.

\section{RESULTS AND DISCUSSION}

This study aims to evaluate quantitative of rational of antibiotic use and qualitative of care for STWs in a university hospital. Results show 13,543 records of STWs. Patients are equally both genders. An average age is 27 years old, about $10 \%$ of patients are over 65 years old. The most two diagnoses are S610: Open wound of finger (s) without damage to nail and W550: Bitten or struck by other mammals at home. On-third of common concomitant disease is E119 (type 2 diabetes mellitus at without complications).

Siriraj hospital, a large university hospital, has been implementing a clinical practice guideline (CPG) for STWs. Class 1 is clean-contaminated wound, normally do not need 
antibiotic prophylaxis because the infection rate is only $5 \%$ or less. It covers smooth border of wound edge, easy to clean, not human or animal bite wound, no exposure of muscle, bone, or tendon, no necrotic tissue, no contamination with feces, urine, saliva, dirt, dirty water, or food ${ }^{5}$. Administration of oral antibiotic at triage also failed to reduce the meaningful reductions in infection rates. The best way to prevent wound infection is thorough wound cleansing and appropriate closure technique ${ }^{7}$. Class 2 wounds and contaminated wounds should receive antibiotics ${ }^{5,7}$. Because this way of classification requires record of chief complaint and physical examination, this research is not able to calculate the percentage of antibiotic use as recommend in RDU manual. We lastly classified ICD-10 codes of STWs into 3 groups; based on details in CPG, available evidence, and expert's opinion. They are group of ICD-10 codes of STWs which need antibiotic, unnecessary antibiotics, and uncertainly need antibiotics.

Table 1. Antibiotics use for STWs from hospital database.

\begin{tabular}{lr}
\hline \multicolumn{1}{c}{ Conditions } & Number of prescription (time) \\
\hline Analysis followed National criteria & 13,566 \\
All STWs cases & 2,549 \\
All STWs cases who prescribed antibiotics & 2,088 \\
\hline Analysis followed Research criteria & 188 \\
STWs who was necessary cases for antibiotics & 5,805 \\
STWs who was necessary cases for antibiotics and had been prescribed antibiotics & 662 \\
STWs who was unnecessary cases for antibiotics & 5,673 \\
STWs who was unnecessary cases for antibiotics and had been prescribed antibiotics & 1,699 \\
STWs who was uncertainly cases for antibiotics & \\
STWs who was uncertainly cases for antibiotics and had been prescribed antibiotics & \\
\hline
\end{tabular}

STWs = Simple Traumatic Wounds

RDU manual determines that STWs cases must prescribed antibiotics less than $40 \%$ of all cases. In the quantitative evaluation, nonrational prescribing in this study is $18.79 \%$ (Table 1). If we exclude necessary cases following expert's opinion, overtreatment with antibiotics in this hospital is only $11.40 \%$. It is acceptable compared with the national target and relatively better than other developing countries ${ }^{3}$.
Antibiotics were used in a lot of ED patients with uncomplicated lacerations despite a lack of evidence for efficacy ${ }^{9}$. This is still leading to increased risk of antimicrobial resistance. Surprisingly, $90.99 \%$ of necessity cases are under treatment with antibiotics. This is leading to potential increased morbidity and mortality. We suggest finding balancing act between under treatment and overtreatment hereafter.

Table 2. Uncertain conditions of antibiotics use for STWs in unnecessary cases.

\begin{tabular}{lc}
\hline \multicolumn{1}{c}{ Conditions } & $\begin{array}{c}\text { Number of prescription } \\
\text { (time) }\end{array}$ \\
\hline Over 65 years old & 912 \\
STWs cases who over 65 years old & 178 \\
STWs cases who over 65 years old and prescribed antibiotics & 334 \\
\hline Concomitant diseases* & 80 \\
STWs cases who has concomitant diseases & \\
STWs cases who has concomitant diseases and prescribed antibiotics & \\
\hline
\end{tabular}

STWs = Simple Traumatic Wound

*Immunocompromised case, Diabetes Mellitus, Cirrhosis, Cancers, HIV

We mention that a number of STWs cases were defined unnecessary for antibiotics. Somehow, we might prescribe antibiotics for uncertain conditions. This study presents only $19.52 \%$ of elderly and $23.95 \%$ of concomitant diseases were prescribed antibiotics (Table 2). It seems leading to deliver under treatment for the risk group. In term of quality of route of administration, this study shows prescription of an antibiotic injection at OPD. In a prescription pattern, ertapenem and meropenem are not appropriate when oral formulations are available. This prescribing practices for antibiotic injection show deviation from the standard recommended by $\mathrm{WHO}^{10,11}$. This finding suggests that antibiotic injection prescribing needs to be regulated. Lastly, we are 
able to explore duration of treatment from quantity of dispensing and the define daily dose (DDD). DDD is an average maintenance dose per day for a drug used for its main indication in adults. Constraint of this estimation is absence of exact regimen in the hospital's database.

Table 3. Number of prescription and medication cost of antibiotics for STWs in all cases.

\begin{tabular}{clrr}
\hline List & Medication cost (Thai baht) & Number of prescription (time) & Medication cost (Thai baht) \\
\hline 1 & Amoxicillin-Clavulanic acid & 2,160 & 181,234 \\
2 & Cephalexin & 204 & 13,298 \\
3 & Clindamycin & 42 & 3,224 \\
4 & Ciprofloxacin & 79 & 1,397 \\
5 & Cefixime & 3 & 804 \\
6 & Ceftriaxone & 13 & 755 \\
7 & Meropenem & 1 & 295 \\
8 & Doxycycline & 25 & 269 \\
9 & Amoxicillin & 5 & 181 \\
10 & Norfloxacin & 5 & 96 \\
11 & Cloxacillin & 5 & 84 \\
12 & Ofloxacin & 4 & 64 \\
13 & Clarithromycin & 2 & 22 \\
14 & Co-trimoxazole & 1 & 6 \\
\hline & & 2,549 & 201,729 \\
\hline
\end{tabular}

STWs $=$ Simple Traumatic Wound

Assessment qualitative of care from treatment regimen is somewhat interesting. Despite amoxycillin is a drug of choice for essential cases, it is prescribed only $0.2 \%$ of all medication.

Amoxycillin and clavulanic acid is the frequently prescribed as same as previous study, even thigh bacterial contaminations of infected patients are usually non-fermentative gramnegative rods, E. cloacae, and mixed organisms ${ }^{12}$ (Table 3). Meanwhile direct costs of antibiotics in Thailand is burden expenditure ${ }^{13}$, this study investigates cost of antibiotics for STWs either. Source of the drugs' price use for cost calculation is retrieved from drugs'cost in the university hospital. In the period 11 months, total antibiotics cost was 5,699,165 Thai baht. Antibiotic cost for STWs takes account for 3.53\%. Based on RDU manual, non-rational use of antibiotics for STWs is $25.48 \%$ of all cases, it costs 57,604 baht yearly. Antibiotics cost for unnecessary STWs cases is double than cost of antibiotics in essential cases (Table 4). As determine conditions in this research, antibiotic cost for STWs in unnecessary cases is 51,409 Thai baht yearly (Table 5). If the hospital strictly controls an antibiotic stewardship and CPG, cost of antibiotics for STWs will reduce.

Limitation of this study is mapping of local drug codes in hospital database (DIDSTD code) and ATC-ID code through E-R diagram in Figure 1. Incomplete relation that crosses boundaries of enable DIDSTD, GP (generic product), TP (trade product), and ATC-ID, is failing to determine the outcome. For example, there is no DIDSTD code of dicloxacillin, we cannot catch ATC-ID. Then we are unable to calculate percentage of antibiotic use based on recommendation in RDU

Table 4. Medication cost of antibiotics for STWs.

\begin{tabular}{|c|c|c|c|}
\hline Conditions & $\begin{array}{l}\text { Medication cost } \\
\text { (Thai baht) }\end{array}$ & $\begin{array}{l}\text { Percentage (\%) of use (compare } \\
\text { with all antibiotics use for STWs) }\end{array}$ & $\begin{array}{c}\text { Percentage (\%) of use } \\
\text { (compare with all antibiotics } \\
\text { use in hospital database) }\end{array}$ \\
\hline $\begin{array}{l}\text { All antibiotics use in } \\
\text { hospital database }\end{array}$ & $5,699,165$ & - & 100.00 \\
\hline Antibiotics use for STWs & 201,729 & 100.00 & 3.53 \\
\hline $\begin{array}{l}\text { Antibiotics use for STWs } \\
\text { in necessary cases }\end{array}$ & 17,261 & 8.56 & 0.30 \\
\hline $\begin{array}{l}\text { Antibiotics use for STWs } \\
\text { in unnecessary cases }\end{array}$ & 51,409 & 25.48 & 0.90 \\
\hline $\begin{array}{l}\text { Antibiotics use for STWs } \\
\text { in uncertain cases }\end{array}$ & 133,059 & 65.96 & 2.33 \\
\hline
\end{tabular}

STWs $=$ Simple Traumatic Wounds 
Table 5. Number of prescription and medication cost of antibiotics for STWs in unnecessary cases.

\begin{tabular}{clcc}
\hline List & Medication cost (Thai baht) & Number of prescription (time) & Medication cost (Thai baht) \\
\hline 1 & Amoxicillin-clavulanic acid & 563 & 45,760 \\
2 & Cephalexin & 49 & 3,446 \\
3 & Clindamycin & 12 & 979 \\
4 & Cefixime & 1 & 440 \\
5 & Ciprofloxacin & 12 & 227 \\
6 & Ceftriaxone & 4 & 216 \\
7 & Doxycycline & 9 & 107 \\
8 & Amoxicillin & 2 & 78 \\
9 & Norfloxacin & 4 & 72 \\
10 & Cloxacillin & 2 & 28 \\
11 & Ofloxacin & 1 & 28 \\
12 & Clarithromycin & 2 & 22 \\
13 & Co-trimoxazole & 1 & 6 \\
\hline & & 662 & 51,409 \\
\hline
\end{tabular}

STWs = Simple Traumatic Wound

manual. We approximately lose $30 \%$ of data in this study.

\section{CONCLUSIONS}

On the basis of the finding of this study, only $18.8 \%$ STWs in ambulatory patients was prescribed antibiotics and it costs 57,604 baht yearly. Follow RDU manual, this result encounters with less than $40 \%$ of antibiotic use in all STWs cases. Follow research criteria, overtreatment STWs with antibiotics in this hospital is only $11.40 \%$. Many STWs cases who are necessity for antibiotics are under treatment. It is leading to potential increased morbidity and mortality. The finding suggests balancing act between under treatment and overtreatment. In term of quality of care, the common drug of choice is amoxicillin and clavulanic acid. It is not adhering with the standard CPG which recommends amoxicillin. Injection of antibiotic at OPD is also improper when oral administration is available. Yearly cost of antibiotics for STWs take account for $3.53 \%$ of all infection diseases. A number of STW cases are under investigation for antibiotics which lead to potential increased morbidity and mortality. In summary, the university hospital has a special responsibility to society to promote rational prescribing by their staffs and, through them, the future generations of doctors. The first concrete recommendations to improve use of medicines is a set of simple indicators for measuring the quality of drug use at the hospital. Drug use evaluation should be done for some of the antibiotics to check whether they were appropriately prescribed or not.

\section{ACKNOWLEDGMENTS}

We sincerely thank all physicians and pharmacists for their help and discussion about criteria and data collection process.

\section{Conflict of interest}

None to declare.

\section{Funding}

None to declared.

\section{Ethics approval}

The study code D-PHA-2561-05365 is exempt for ethic allowance from the ethical review committee for human research, Faculty of medicine, Chiangmai University on January 17, 2019.

\section{Article info:}

Received December 30, 2019

Received in revised form May 9, 2020

Accepted July 26, 2020

\section{REFERENCES}

1. Ofori-Asenso R, Agyeman A. Irrational use of medicines a summary of Key concepts. Pharmacy. 2016;4(4):35.

2. Chayakul C, Jongtrakul P, Wananukul W, Punnupurot $\mathrm{P}$, Kanjanarat P. Yotsombat K, et al. Rational drug use manual for rational drug use in health promotion hospital. Bangkok: Agriculture co-operative federation of Thailand Publishing. 2015.

3. Laing RO, Hogerzeil HV. Ten recommendations to improve use of medicines in developing countries. Health Policy Plan. 2001;16(1):13-20.

4. Chalortham N, Kiewchaum T, Laophokhin V, Kitikannakorn N. Development of BC2017 Tool to Process KPIs Information of Antibiotic Smart Use In the $15^{\text {th }}$ Health Service Plan. TJPP. 2018;10(2):392-401.

5. Sirijatuphat R, Choochan $T$, Siritongtaworn $P$, Sripojtham V, Thamlikitkul V. Implementation of antibiotic use guidelines for fresh traumatic wound at Siriraj Hospital. J Med Assoc Thai. 2015; 
98(3): 245-52.

6. Otterness K, Singer AJ. Updates in emergency department laceration management. Clin Exp Emerg Med. 2019;6(2):97-105.

7. Prevaldi C, Paolillo C, Locatelli C, Ricci G, Catena F, Ansaloni L, et al. Management of traumatic wounds in the Emergency Department: position paper from the Academy of Emergency Medicine and Care (AcEMC) and the World Society of Emergency Surgery (WSES). World J Emerg Surg. 2016;11:30.

8. Ong S, Moran GJ, Krishnadasan A, Talan D. Antibiotic Prescribing Practices of Emergency Physicians and Patient Expectations for Uncomplicated Lacerations. West J Emerg Med. 2011;12(4):375-80.

9. Cummings P, Del Beccaro MA. Antibiotics to prevent infection of simple wounds: a meta-analysis of randomized studies. Am J Emerg Med. 1995;13(4):396-400.

10. Desalegn AA. Assessment of drug use pattern using WHO prescribing indicators at Hawassa University teaching and referral hospital, south Ethiopia: a crosssectional study. BMC Health Serv Res. 2013;13:2-6.

9. Cowell DL, Harvey M, Cave G. Antibiotic prophylaxis at triage for simple traumatic wounds: a pilot study. Eur J Emerg Med. 2011;18(5):279-81.

11. Bilal AI, Osman ED, Mulugeta A. Assessment of medicines use pattern using World Health Organization's prescribing, patient care and health facility indicators in selected health facilities in eastern Ethiopia. BMC Health Serv Res. 2016;16:144.

12. Sirijatuphat R, Siritongtaworn P, Sripojtham V, Boonyasiri A, Thamlikitkul V. Bacterial contamination of fresh traumatic wounds at trauma center, Siriraj Hospital, Bangkok, Thailand. J Med Assoc Thai. 2014;97 Suppl 3:S20-5.

13. Thamlikitkul V. Final Report on Phase I Thailand Antimicrobial Resistance Containment and Prevention Program. Thai Health Promotion Foundation and Health Systems Research Institute (Thailand), 2017. 